\title{
Robust Path Design Algorithms for Traffic Engineering with Restoration in MPLS Networks*
}

\author{
Emre Yetginer and Ezhan Karasan \\ Department of Electrical and Electronics Engineering \\ Bilkent University \\ Bilkent 06533, Ankara, Turkey \\ \{yetginer,ezhan\}@ee.bilkent.edu.tr
}

\begin{abstract}
In this paper we study traffic engineering of restorable paths in Multiprotocol Label Switching (MPLS) networks. We consider off-line computation of working and restoration paths with path rerouting as the restoration scheme. First we compute a link-disjoint path set for given set of demands. Using this path set we study four approaches for selecting working and restoration paths, and formulate each method as an Integer Linear Programming (ILP) problem. A traffic uncertainty model is developed in order to compare these approaches based on their robustness with respect to changing traffic patterns. We obtain numerical results and compare these design approaches based on the number of additional demands carried and the distribution of residual capacity over the network.
\end{abstract}

\section{Introduction}

Multiprotocol Label Switching (MPLS) is an advanced forwarding technology which uses the control plane of the IP routing protocol. Packets in the network are divided into subsets called Forwarding Equivalence Classes (FEC) based on their source and destination IP addresses, network and transport layer protocol sources. The main idea of MPLS is to map the packets to a FEC at the entry into an MPLS domain (called ingress router) and use only FEC based labels to process and forward these packets inside the domain. The routing is done at the ingress point and capacities along the selected route are reserved. All forwarding in an MPLS domain is done only by using the data contained in the label, resulting in an increase in forwarding speed. The label is removed at the exit point of the MPLS domain

\footnotetext{
${ }^{*}$ Work partially supported by the Scientific and Technological Research Council of Turkey (TUBITAK) under the project 199E005.
}

(called egress router). MPLS architecture is described in detail in $[1,2]$.

With MPLS it is possible to exercise traffic engineering in order to have efficient use of network resources by forcing some traffic to follow an explicitly specified path in order to avoid congested parts of the network. Traffic engineering problem is discussed in [3], and the key aspects of MPLS that can be used to solve this problem are emphasized.

MPLS can react rapidly to faults by switching failed connections with higher QoS requirements to secondary paths. General specifications and bandwidth reservation for protection are discussed in [4]. Providing reliable services in MPLS is studied and fast rerouting techniques are proposed in the Internet draft [5]. New algorithms for dynamic routing of restorable bandwidth guaranteed paths are presented and formulated in [6].

In this paper, we study the traffic engineering problem for MPLS networks which are fully restorable against all single-link failures. We assume that the MPLS network has a 2-connected topology with given link capacities. An initial traffic demand set is given which is obtained based on some traffic forecasts. Using this demand set, we design working and restoration paths for all requested connections subject to the constraints that the traffic on each link does not exceed its capacity, and all requested connections can be fully restored against all possible single-link failures. After routes are designed, the network is subjected to additional traffic demand which models the uncertainty in the forecasts. The main goal is to carry as many of additional requests as possible subject to the constraints that all existing working paths remain unchanged, all link capacity constraints are satisfied, and all carried traffic in the network is fully restorable.

In order to increase the number of carried additional connections, we reoptimize restoration paths for existing connections. The goal of the traffic engineering process is to 
provide spare capacity on all links so that a larger number of additional demands are carried. We develop design algorithms which distribute unused capacity over the network in such a way that links with higher likelihood of carried additional traffic are assigned larger spare capacity. These algorithms are formulated as Integer Linear Programming (ILP) problems, and the performance of these algorithms are compared through numerical examples.

In this work, a restoration scheme with off-line path rerouting with resource reservation is studied. We discuss four different methods for solving the network design problem. First two methods treat the problems of designing working and restoration paths separately. And the last two methods jointly optimize the working and restoration path design problems. A traffic uncertainty model is introduced which is used to evaluate relative performances of these four network design algorithms.

\section{Separate Design Of Working And Restora- tion Paths with Minimum Bandwidth Us- age}

We first compute a path set for given demands. The path set consists of all possible link-disjoint paths for each demand such that the capacity usage is minimized. Computation of the path set for a demand is accomplished in two steps. The maximum number of link-disjoint paths between the source and destination nodes, which is denoted by $D$, can be obtained from max-flow problem formulation [7]. While we want to compute $D$, we would also like to find these link-disjoint paths such that they have the minimum total capacity usage. We combine these two parts and formulate the overall problem as a single ILP. The set of all paths obtained at the end of this computation is denoted by $P=\left\{P_{k i}\right\}$, where $P_{k i}$ is the $i^{t h}$ path for demand $k$.

In the separate path design approach, the problems of designing working and restoration paths are treated sequentially. The goal in designing the working and restoration paths is to minimize the total capacity used in the network. First, working paths are chosen to minimize the total working capacity used in the network while satisfying all demands. ILP formulation for this problem is given by

$$
\begin{aligned}
& \max \sum_{l} z_{l} \\
& \text { subject to } \\
& \sum_{i} x_{k i}=1, \forall k \\
& \sum_{k} \sum_{i} x_{k i} r_{k} \delta_{k i}^{l}+z_{l} \leq C_{l}, \quad \forall l \\
& x_{k i} \in\{0,1\}, \quad z_{l} \geq 0
\end{aligned}
$$

where $x_{k i}$ is the decision variable defined as $x_{k i}=1$, if $P_{k i}$ is chosen as working path for demand $k$, and $x_{k i}=0$, otherwise. The other decision variable $z_{l}$ denotes the amount of residual capacity on link $l$. The input parameter $r_{k}$ is the bandwidth requested by demand $k, C_{l}$ is the capacity of link $l$, and $\delta_{k i}^{l}$ is the path-link incidence indicator defined as $\delta_{k i}^{l}=1$, if $P_{k i}$ passes through link $l$, and $\delta_{k i}^{l}=0$, otherwise. The objective in the above ILP formulation is to maximize the total residual capacity in the network after all demands are routed. The first constraint implies that all demands are satisfied. The second constraint is the link capacity constraint which ensures that the total capacity used on link $l$ does not exceed $C_{l}$. The solution of this problem gives the selected path for each demand and the residual capacity on each link after all demands are routed.

Next, restoration paths are chosen such that the total unused capacity in the network is maximized after restoration paths are assigned for all demands. The set of all possible restoration paths, $P^{*}$, is obtained by excluding all selected working paths from the path set $P$. In other words, path set $P^{*}$ is obtained by deleting the paths chosen as working paths from $P$. The difference in the design of the restoration paths is that the capacity reserved for restoration on a link can be shared by working paths that are link-disjoint since only single-link failures are considered. The ILP formulation for the design of restoration paths is given by

$$
\begin{aligned}
& \max \sum_{l} z_{l} \\
& \text { subject to } \\
& \sum_{i} y_{k i}=1, \forall k \\
& \sum_{k} \sum_{i} y_{k i} r_{k} \varepsilon_{l k i l^{\prime}}+z_{l^{\prime}} \leq C_{l^{\prime}}^{*}, \forall l, \forall l^{\prime} \\
& y_{k i} \in\{0,1\}, \quad z_{l} \geq 0
\end{aligned}
$$

where the decision variable $y_{k i}$ is defined as $y_{k i}=1$, if $P_{k i}^{*}$ is chosen as restoration path for demand $k$, and $y_{k i}=0$, otherwise. $z_{l}$ is the residual capacity on link $l$. The indicator function $\varepsilon_{l k i l^{\prime}}$ is defined as $\varepsilon_{l k i l^{\prime}}=1$, if $k^{\text {th }}$ demand uses link $l$ on its active path and $i^{t h}$ backup path uses link $l^{\prime}$, and $\varepsilon_{l k i l^{\prime}}=0$, otherwise. In this formulation the capacity of link $l, C_{l}^{*}$, is obtained after $C_{l}$ is decreased by the total capacity used by working paths on link $l$. The objective is again to maximize the total residual capacity which is the sum of residual capacities on all links. The first constraint ensures that a restoration path is selected for each demand. The second constraint is the capacity constraint which inherently takes into account possible sharing of capacity between different restoration paths. This constraint states that in case of failure of link $l$, bandwidth used on each link does not exceed its available capacity.

Separate design of working and restoration paths results in the minimum capacity usage for working and restoration 
paths. One possible disadvantage of this approach is that the network capacity may be used in an unbalanced manner where some links may be congested while other links are underutilized. As a result, the residual network may face problems with routing additional demands which are introduced later into the network as the traffic demand increases.

\section{Separate Design Of Working And Restora- tion Paths With Load Balancing}

One possible solution for avoiding this uneven distribution of network load is considered in this section. The idea is to distribute the load for working paths in a such a fashion that at least some certain amount of unused capacity remains on each link. Then the restoration paths can be computed in a similar manner on the residual network. More formally, the minimum residual capacity, where the minimum is taken over all links, is maximized separately for both working and restoration path design problems. Thus a two step optimization is employed for working and restoration path design problems similar to the previous method.

In the first stage, working paths are designed such that the minimum residual capacity is maximized. The ILP formulation for the first step is as follows.

$$
\begin{aligned}
& \max z+\alpha \sum_{l} z_{l} \\
& \text { subject to } \\
& \sum_{i} x_{k i}=1, \quad \forall k \\
& \sum_{k} \sum_{i} x_{k i} r_{k} \delta_{k i}^{l}+z_{l} \leq C_{l}, \quad \forall l \\
& z \leq z_{l}, \forall l \\
& x_{k i} \in\{0,1\}, \quad z \geq 0, \quad z_{l} \geq 0
\end{aligned}
$$

where $x_{k i}$ is the decision variable defined as $x_{k i}=1$, if $P_{k i}$ is chosen as working path for demand $k$, and $x_{k i}=0$, otherwise. $z_{l}$ is the residual capacity on link $l, z$ denotes the minimum residual capacity, and $\delta_{k i}^{l}$ is the path-link incidence indicator function defined as $\delta_{k i}^{l}=1$, if $P_{k i}$ passes through link $l$, and $\delta_{k i}^{l}=0$, otherwise.

The objective is to maximize the minimum residual capacity while simultaneously maximizing the total residual capacity in the network. The parameter $\alpha$ is chosen very small such that, the maximization of $z$ has higher priority. The first constraint ensures that for all demands exactly one working path is chosen. The second constraint states that the bandwidth used on each link does not exceed the capacity of that link. And the last constraint is used to set $z$ to the minimum of the residual link capacities. The solution for this problem determines working paths for all demands.

Restoration paths are selected in a similar way. The path set $P$ is updated so that the selected working paths for each demand are discarded, and a reduced path set, $P^{*}$, is obtained. And the capacity of each link is reduced by the total capacity used by all working paths on that link, so the set of modified link capacities, $\left\{C_{l}^{*}\right\}$, is obtained. The ILP formulation for designing the restoration paths is given as

$$
\begin{aligned}
& \max z+\alpha \sum_{l} z_{l} \\
& \text { subject to } \\
& \sum_{i} y_{k i}=1, \quad \forall k \\
& \sum_{k} \sum_{i} y_{k i} r_{k} \varepsilon_{l k i l^{\prime}}+z_{l^{\prime}} \leq C_{l^{\prime}}^{*}, \forall l, \forall l^{\prime} \\
& z \leq z_{l}, \forall l \\
& y_{k i} \in\{0,1\}, z \geq 0, z_{l} \geq 0
\end{aligned}
$$

where the decision variable $y_{k i}$ is defined as $y_{k i}=1$, if $P_{k i}^{*}$ is chosen as restoration path for demand $k$, and $y_{k i}=0$, otherwise. $z_{l}$ is the residual capacity on link $l$, and $z$ denotes the minimum residual capacity. The indicator function $\varepsilon_{l k i l^{\prime}}$ is defined as $\varepsilon_{l k i l^{\prime}}=1$, if the working path for $k$-th demand uses link $l$ and $P_{k i}^{*}$ uses $\operatorname{link} l^{\prime}$, and $\varepsilon_{l k i l^{\prime}}=0$, otherwise.

The objective is to maximize the minimum residual capacity while simultaneously maximizing the total residual capacity in the network. The first constraint states that for each demand only one path is chosen as the restoration path. The second constraint is the capacity constraint which ensures that in case of failure of link $l$, the restoration capacity used on each link $l^{\prime}$ does not exceed the capacity $C_{l^{\prime}}^{*}$. And the last constraint sets $z$ to the minimum of the residual capacities. As a result, restoration paths for all demands are selected in a way that balances residual capacities on all links.

\section{Joint Design Of Working And Restoration Paths With Load Balancing}

Both design methods described in Sections 2 and 3 solve the working and restoration paths design problems separately. But it is clear that the two problems interact with each other. The design of restoration paths can be more efficient if the working paths are designed such that maximum sharing between restoration paths is obtained. The design methods of Sections 2 and 3 try to minimize the capacity used for working and restoration paths independently. This does not guarantee that the total used bandwidth is minimized. The reason is that by designing working paths in a suitable manner, possibly using more bandwidth, the capacity needed for restoration paths can be reduced. Thus the total used capacity for working and restoration paths can be less than the separate design models. In this section a design 
method is introduced that jointly optimizes the working and restoration paths with load balancing. The ILP formulation for this method is given as

$$
\begin{aligned}
& \max z+\alpha \sum_{l} z_{l} \\
& \text { subject to } \\
& \sum_{i} \sum_{j} v_{k i j}=1, \forall k \\
& v_{k i j}=0, \text { if } i=j, \forall i, j, k \\
& \sum_{k} \sum_{i} \sum_{j} v_{k i j} r_{k} \delta_{k i}^{l^{\prime}}+\sum_{k} \sum_{i} \sum_{j} v_{k i j} r_{k} \delta_{k j}^{l^{\prime}} \delta_{k i}^{l} \\
& \qquad+z_{l^{\prime}} \leq C_{l^{\prime}}, \forall l, \forall l^{\prime} \\
& z \leq z_{l}, \forall l \quad \\
& v_{k i j} \in\{0,1\}, z \geq 0, z_{l} \geq 0
\end{aligned}
$$

where $\delta_{k i}^{l}$ is the indicator function defined as $\delta_{k i}^{l}=1$, if $P_{k i}$ uses link $l$, and $\delta_{k i}^{l}=0$, otherwise. The decision variable $v_{k i j}$ is defined as $v_{k i j}=1$, if $P_{k i}$ and $P_{k j}$ are choosen as working and restoration paths, respectively, for demand $k$, and $v_{k i j}=0$, otherwise. $z_{l}$ is the residual capacity on link $l$, and $z$ denotes the minimum residual capacity.

The objective is to maximize the minimum residual capacity while simultaneously maximizing the total residual capacity in the network so that the residual capacity is distributed uniformly and efficiently. In the objective function, the parameter $\alpha$ is chosen small so that the maximization of $z$ takes the higher priority. First constraint ensures that one working and one restoration path is chosen for each demand. Second constraint states that the same path cannot be chosen as both working and restoration path for any demand. Third constraint is the capacity constraint on link $l^{\prime}$ stating that in the case of failure of link $l$, the capacity used for working and restoration paths on any other link $l^{\prime}$ cannot exceed its capacity $C_{l^{\prime}}$. The last constraint sets $z$ to the minimum of the residual link capacities.

\subsection{Joint Design of Working and Restoration Paths with Weighted Load Balancing}

This design approach is similar to the joint optimization formulation given above. The difference is that the constraint stated in (1) is replaced by $z \leq \omega_{l} z_{l}$, where $\omega_{l}$ denotes the relative weight of link $l$. In the case where all weights are equal to unity, as in the previous method, the residual capacity is uniformly distributed over the network, neglecting the relative importance of each link. This approach may cause some links to become bottlenecks since the link capacity utilizations vary depending on the network topology and traffic distribution. It may be a better design approach to have more residual capacities on links that are candidates of being overloaded, i.e., links with high estimated utilization levels. This is accomplished by assigning each link a weight which is inversely proportional with the estimated utilization level on that link. The links with high probability of usage are given less weight, so that by maximizing the minimum of the weighted residual capacities these links will have more residual capacities.

\section{Traffic Uncertainity Modeling}

The demands on a network are not deterministic quantities. They are typically obtained from some traffic measurements and forecasts, and link capacities are designed based on these traffic projections. Link capacities are expanded typically every few years in order to cope up with increasing traffic demand and to relieve bottlenecks occurring as a result of deviations from traffic projections. Hence, there is always an uncertainty in the demand structure. An important performance measure of any methodology for designing working and restoration paths is its robustness against traffic uncertainty. The designed network should be able to delay the trivial and expensive solution of capacity expansion as much as possible by efficiently using the available capacity.

To compare the relative efficiencies of the four methods presented in this paper, traffic uncertainty is modeled as additional demands on top of the given demands. We compare different design approaches by calculating the number of additional demands that can be carried for each design. In all methods designed working paths are not allowed to be reconfigured in order to minimize the effect of reconfiguration on existing traffic. But restoration paths for existing demands can be reoptimized in order to maximize the number of carried new connection requests. The performance measure for each method is defined as the number of additional demands the network can carry under that design.

The ILP formulation for traffic uncertainty model is given below. The subscript $k$ is used for existing demands, and $k_{e}$ is used to denote additional demands. The path set $P$ is updated so that the working paths for existing demands are discarded, and the reduced path set $P^{*}$ is obtained. $P^{e}$ is the path set for additional demands. The capacity of each link is reduced by the total bandwidth used by all existing working paths on that link, so the set of modified link capacities, $\left\{C_{l}^{*}\right\}$, is obtained.

$$
\begin{aligned}
& \max \sum_{k_{e}} \sum_{i} \sum_{j} v_{k_{e} i j} \\
& \text { subject to } \\
& \sum_{i} y_{k i}=1, \forall k \\
& v_{k_{e} i j}=0, \text { if } i=j, \forall i, j, k_{e}
\end{aligned}
$$




$$
\begin{aligned}
& \sum_{i} \sum_{j} v_{k_{e} i j} \leq 1, \forall k_{e} \\
& \sum_{k_{e}} \sum_{i} \sum_{j} v_{k_{e} i j} r_{k_{e}} \delta_{k_{e} i}^{l^{\prime}}+\sum_{k_{e}} \sum_{i} \sum_{j} v_{k_{e} i j} r_{k_{e}} \delta_{k_{e} j}^{l^{\prime}} \delta_{k_{e} i}^{l} \\
& \quad+\sum_{k} \sum_{i} \varepsilon_{l k i l^{\prime}} y_{k i} r_{k} \leq C_{l^{\prime}}^{*}, \forall l, \forall l^{\prime} \\
& v_{k_{e} i j} \in\{0,1\}, y_{k i} \in\{0,1\}
\end{aligned}
$$

where $v_{k_{e} i j}$ is the decision variable defined as $v_{k_{e} i j}=1$, if $P_{k_{e} i}^{e}$ and $P_{k_{e} j}^{e}$ are chosen as working and restoration paths, respectively, for new demand $k_{e}$. The other decision variable $y_{k i}$ denotes the restoration path chosen for existing demand $k$, which is defined as $y_{k i}=1$, if $P_{k i}^{*}$ is chosen as restoration path for demand $k$, and $y_{k i}=0$, otherwise. The indicator function $\delta_{k i}^{l}$ is the path-link incidence function defined as $\delta_{k_{e} i}^{l}=1$, if $P_{k_{e} i}^{e}$ uses link $l$, and $\delta_{k_{e} i}^{l}=0$, otherwise. The indicator function $\varepsilon_{l k i l^{\prime}}$ is defined as $\varepsilon_{l k i l^{\prime}}=1$, if the existing working path for $k^{t h}$ demand uses link $l$ and $P_{k i}^{*}$ uses link $l^{\prime}$.

The objective is to maximize the number of additional demands that can be carried. The first constraint ensures that a restoration path is selected for each existing demand. The second constraint states that separate working and restoration paths are chosen for each additional demand. The third constraint ensures that at most one working and restoration path pair is chosen for each additional demand $k_{e}$. The last constraint is the capacity constraint for link $l^{\prime}$ stating that in case of failure of any link $l$ the capacity constraint on link $l^{\prime}$ is not violated. The first term on the left-hand side is the necessary capacity for working paths on link $l^{\prime}$ corresponding to additional demands, and the second and the third terms are the restoration capacities required for additional and existing demands respectively, in case of failure of link $l$.

\section{Numerical Results}

In this section, numerical results for the working and restoration paths design methods are presented. A mesh network with 32 nodes and 50 links with given link capacities is considered. Each demand set consists of $80 \mathrm{de}-$ mands with randomly chosen source-destination nodes and bandwidth requirements. Bandwidth requirement for each demand is selected randomly from the set $\{1,2,3\}$, and a path set is created for the given demand set. The working and restoration paths are designed for all demands with the four methods presented in this paper. Results are obtained for ten different sets of demands. Method 1 to Method 4 correspond to Separate Design of Working and Restoration Paths, Separate Design of Working and Restoration Paths with Load Balancing, Joint Design of Working and Restoration Paths with Load Balancing and Joint Design of Work-

\begin{tabular}{||c||c|c|c||}
\hline \hline \multirow{2}{*}{ Method } & \multicolumn{3}{c||}{ Demand Set 1 } \\
\cline { 2 - 4 } & Working & Restoration & Residual \\
\hline \hline 1 & 689 & 505 & 528 \\
\hline 2 & 694 & 513 & 515 \\
\hline 3 & 701 & 416 & 605 \\
\hline 4 & 708 & 409 & 605 \\
\hline \hline
\end{tabular}

Table 1. Network capacity usage for demand set 1

\begin{tabular}{||c||c|c|c||}
\hline \hline \multirow{2}{*}{ Method } & \multicolumn{3}{|c||}{ Demand Set 1 } \\
\cline { 2 - 4 } & Working & Restoration & Residual \\
\hline \hline 1 & 687 & 495 & 540 \\
\hline 2 & 706 & 456 & 560 \\
\hline 3 & 714 & 417 & 591 \\
\hline 4 & 709 & 414 & 599 \\
\hline \hline
\end{tabular}

\section{Table 2. Network capacity usage for demand set 2}

ing and Restoration Paths with Weighted Load Balancing methods, respectively.

Tables 1 and 2 show total network capacity used by demand sets 1 and 2 for all four design techniques. For the first demand set, although total working capacity used is minimized with method 1 , it increases only slightly with other methods. But the restoration capacities reserved are much lower with the last two methods resulting in more residual capacity in the network. This result shows the strong dependence of the working and restoration path design problems. Similar results are observed for the second demand set.

The distribution of residual capacity over the network is as important as the amount of total residual capacity. Figure 1 shows residual capacity distributions over the links for demand set 1 . Plots on the first row show the number of links with residual capacity given on the horizontal axis. The residual capacity ratio is defined as the ratio of residual capacity to link capacity. Plots on the second row show the number of links having a residual capacity ratio given on the horizontal axis. It is seen that the first method results in larger number of links with small residual capacities. With the load balancing approach of the second method, the number of links with small residual capacities decreases. Thus a better distribution of residual capacity over the network is obtained. Results for methods 3 and 4 show much better distributions, since the number of links with high utilization are smaller than the first two techniques. Besides, the minimum residual capacity also increases in the last two methods eliminating possible bottlenecks in the network. 
Still another observation is that, while the third method distributes the residual capacity evenly across the network, the last method balances the utilization levels on the links more uniformly.
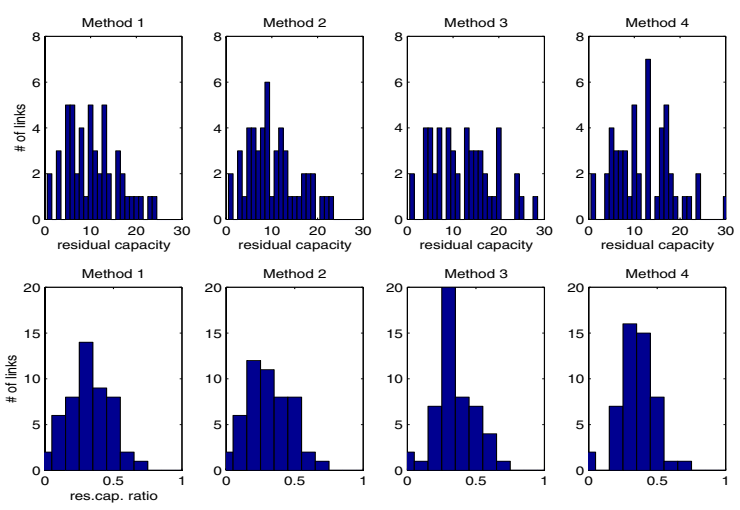

\section{Figure 1. Residual capacity distribution for} demand set 1

To compare relative performances of each design approach, the traffic uncertainty modeling described in Section $\mathrm{V}$ is used. For each demand set of existing traffic, 20 sets of randomly chosen additional demands are determined similar to the original demands. The average number of additional demands that can be routed over the designed networks with each method is tabulated in Table 3 . The number of additional demands is 25 for the first two demand sets, and there are 20 additional demands for other demand sets. With nearly the same residual capacity the last method is more successful in carrying additional demands than the third approach. Except for one case, the worst performance is obtained with the first method. Load balancing feature of the second method improves the performance by a significant amount. And the joint optimization method increases the number of additional demands that can be carried. Further improvement is obtained by the weighted load balancing approach of the last method. These results demonstrate the importance of the distribution of the residual capacity in the network in order to realize robust path design.

\section{Conclusion}

MPLS is a switching technology that presents advantages of traffic engineering and QoS support on IP networks. Moreover, fast restoration capability of MPLS networks may meet increasing need of reliability in the Internet. In this context appropriate design of networks to efficiently utilize resources is a critical problem. In this paper, we study the problem of designing working and restoration paths in a robust way, and present four design approaches.

\begin{tabular}{||c|c|c|c|c||}
\hline \hline \multirow{2}{*}{$\begin{array}{c}\text { Demand } \\
\text { set }\end{array}$} & \multicolumn{4}{|c||}{ Average \# of carried demands } \\
\cline { 2 - 5 } & Met. \#1 & Met. \#2 & Met. \#3 & Met. \#4 \\
\hline \hline 1 & 19.45 & 20.15 & 21.55 & 21.80 \\
\hline 2 & 19.80 & 20.70 & 21.75 & 22.20 \\
\hline 3 & 18.75 & 19.10 & 19.55 & 19.55 \\
\hline 4 & 18.10 & 18.50 & 19.00 & 18.75 \\
\hline 5 & 18.05 & 18.00 & 18.60 & 18.65 \\
\hline 6 & 17.25 & 17.95 & 17.95 & 18.10 \\
\hline 7 & 16.50 & 18.10 & 18.00 & 18.40 \\
\hline 8 & 18.85 & 19.60 & 19.65 & 19.65 \\
\hline 9 & 17.20 & 17.35 & 19.05 & 19.35 \\
\hline 10 & 17.55 & 18.60 & 18.60 & 18.75 \\
\hline \hline
\end{tabular}

Table 3. Average number of additional demands carried with four path design methods

We develop a traffic uncertainty model to compare relative performances of these methods. We show that by carefully distributing the traffic load over network resources the joint design approach with weighted load balancing perform better than other design approaches in carrying additional traffic resulting from traffic uncertainty. The problem of extending these robust path design methods to Multi-Protocol Lambda Switching (MP $\lambda \mathrm{S}$ ) networks where optical transmission impairments place additional constraints on path set selection is currently under investigation.

\section{References}

[1] E. Rosen, A. Viswanathan, and R. Callon, "Multiprotocol Label Switching Architecture", Internet Draft draftietf-mpls-arch-07.txt, July 2000.

[2] B. Davie, Y. Rekhter, MPLS Technology and Applications, Morgan Kaufmann, 2000.

[3] G. Swallow, "MPLS Advantages for Traffic Engineering”, IEEE Comm. Mag., Dec 1999, pp.54-57.

[4] Thomas M. Chan, "Reliable Services in MPLS", IEEE Comm. Mag., Dec 1999, pp. 58-62.

[5] L. Mo, "General Considerations for Bandwidth Reservation in Protection", Internet Draft draft-mo-mplsprotection-00.txt, July 2000.

[6] M.Kodialam and T.V. Lakshman, "Dynamic Routing of Bandwidth Guaranteed Tunnels with Restoration", IEEE Infocom 2000.

[7] R. K. Ahuja, T. L. Magnanti and J. B. Orlin, Network Flows. Englewood Cliffs, NJ: Prentice Hall, 1993. 